\title{
TESL Methodology in Canada: A Study of Instructor Attitudes and Correlates
}

\author{
Gary Libben \\ Tammi Rossman-Benjamin
}

This paper reports on a survey of ESL teachers' attitudes toward classroom techniques. Sixty-four teachers, sampled from 5 ESL centres across Canada, completed a 95-item questionnaire which investigated attitudes toward specific ESL techniques and the factors which might influence them. The results indicated that teachers' age, amount and type of TESL experience, and individual learning style are all related to TESL attitudes. These effects, however, were localized to particular clusters of attitudes.
The characteristics of an instructor's teacher-training course, on the other hand, affected the entire range of responses. A correlation of .85 was found between ESL teachers' attitudes and the methodological attitudes expressed by their TESL teachers during teacher-training. We conclude that the attitudes of both the ESL and TESL teachers are influenced by a set of "cultural norms and values" in which all members of the TESL community participate.

The teaching of English as a second language in Canada is not what it was a decade ago. The field has passed through a period of professionalization which has resulted in better trained teachers, more professional journals and a proliferation of provincial and local TESL organizations. Major presses have published books on ESL methods and approaches (see in particular Larsen-Freeman (1986); Richards and Rodgers (1986)) and ESL teachers generally enjoy greater freedom of approach both in the classroom and at the bookstore.

What are the consequences of this methodological freedom? Do Canadian ESL teachers have a shared set of views on effective classroom practices or has the ESL teacher been individualized with respect to methodology?

The goal of this study was to address the above question by investigating teaching style in Canadian adult ESL classrooms and the factors which may influence a teacher's methodological preferences. To this end we developed a teaching style questionnaire which consists of 95 questions. The questionnaire was distributed to ESL teachers at 5 adult ESL centres in 4 Canadian cities (Montreal, Cornwall, Calgary, and Edmonton). ${ }^{1} \quad$ The sample from these centres consists of 64 respondents. 
Our report is divided into four parts. Immediately below, we discuss the organization and content of the questionnaire. In Part 2, we describe the attitudes toward methods, techniques and language skills that were elicited by the questionnaire. In Part 3, we present our analysis of the relationship between these attitudes and factors such as teacher age, teaching experience, cognitive style and teacher-training experience. Finally, in Part 4 , the implications of the study are discussed. In this section we present the hypothesis that teachers' attitudes are best understood in terms of a model of TESL Culture. This is a restricted "culture" in the sense that it is almost exclusively concerned with matters related to teaching and learning. We argue, however, that it is a culture nonetheless-characteristically exhibiting a shared set of values which members consider to be self-evident, which no member knows the source of, and which every member considers to be the product of his or her own conscience.

\section{PART 1: THE TEACHING STYLE QUESTIONNAIRE}

\section{Attitudes toward specific ESL techniques and practices}

As can be seen in Figure 1, the teaching style questionnaire is organized around the measurement of teachers' attitudes toward ESL methods, techniques and the relative importance of language skills. The measurement of TESL attitudes rather than TESL behaviour in this research allowed us to elicit responses which were not dependent on the orientation and requirements of particular institutions and programs within which the teachers were working. All respondents were asked to make judgements on the basis of what they considered to be desirable classroom procedure for a group of intermediate adult ESL students all of whom speak the same mother tongue. Thus it was possible for us to elicit teachers' views on the use of translation in the ESL classroom with respect to a scenario in which such a practice would be possible (i.e., one in which all students have the same mother tongue).

The major component of the attitude survey is a section in which teachers are presented with a set of 23 statements concerning desirable and undesirable TESL activities. The respondents were required to rate these statements on a five-point scale ranging from "strongly agree" to "strongly disagree". An example of the format of this section is provided in Figure 2.

Note that the examples in Figure 2 contain positively and negatively framed questions. This section of the questionnaire 


\section{FIGURE 1}

The organization of the attitude survey. Variables which were hypothesized to influence attitudes are represented in peripheral ovals.

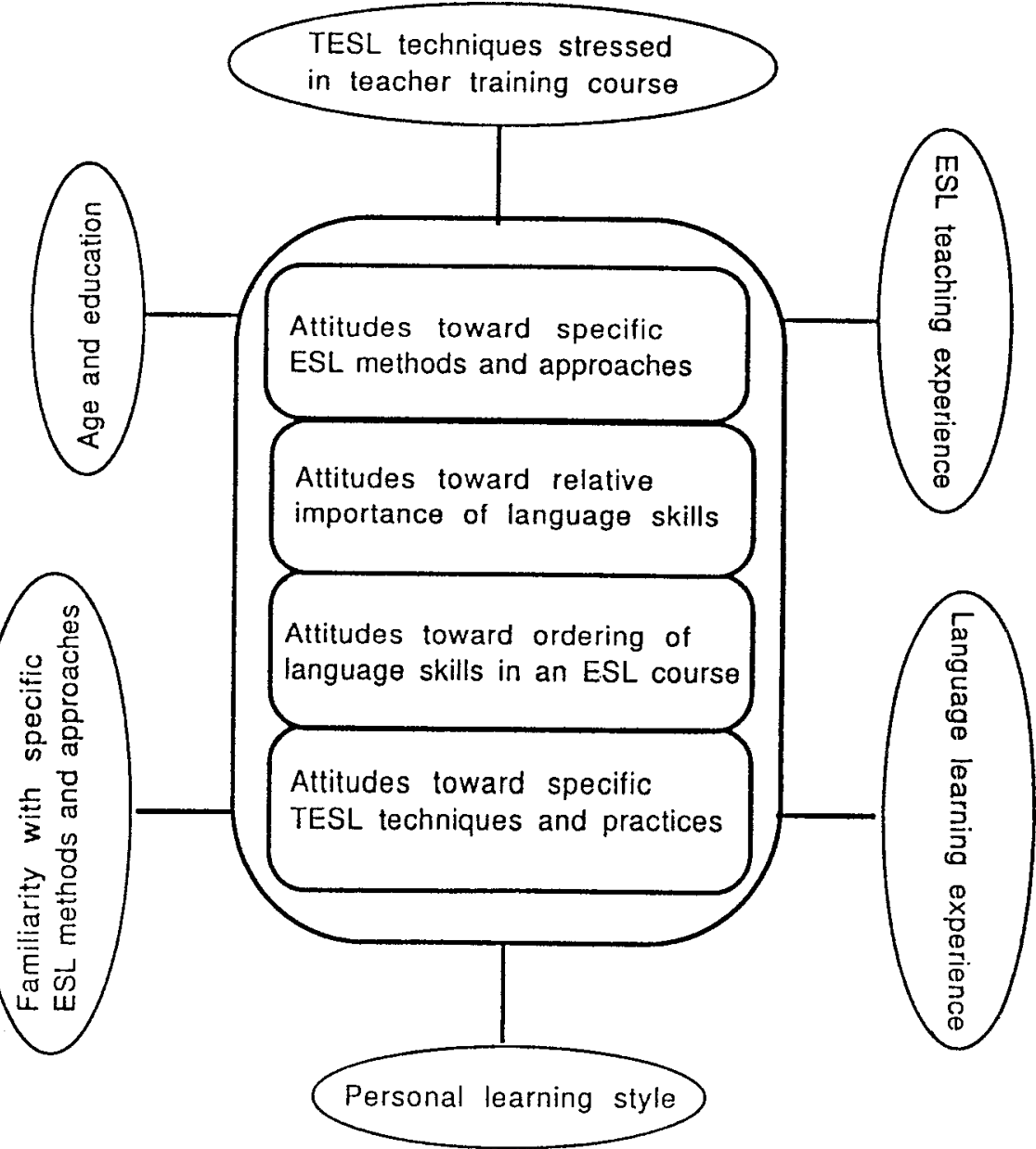

contains 20 questions which are organized around a set of teaching style "distinctive features". Each feature is represented by two statements-one positive and one negative. For example, item 3 in Figure 2 has as its complement the following item:

17. Having students translate from their mother tongue to English or the other way around can be an important pedagogical tool.

Both items 3 and 17 are instances of the feature "translation". 
FIGURE 2

The first five items of the Technique Attitudes section of the questionnaire.

1. A good teacher should correct his or her students' grammatical errors in their speech.

strongly agree agree neutral disagree strongly disagree

2. The teacher can help the student by providing her or him with explicit rules of English syntax.

strongly agree agree neutral disagree strongly disagree

3. It is poor practice to give students exercises that require them to translate from their mother tongue to English or the other way around.

strongly agree agree neutral disagree stmongly disagree

4. The teacher's use of the mother tongue in the class is a detriment to learning another language.

strongly agree agree neutral disagree strongly disagree

5. A good teacher should not rely solely on one method or textbook no matter how well-organized it may be.

strongly agree agree neutral disagree strongly disagree

The entire set of features plus three items which poll teachers' attitudes toward the correction of students' errors in the domains of pronunciation, written syntax, and oral syntax are given below:

$\begin{array}{lll}\text { F1. } & \text { CONTRAST } & \text { (contrasting English and students' mother tongue) } \\ \text { F2. } & \text { ECLECTIC } & \text { (not adhering to a single method or textbook) } \\ \text { F3. } & \text { PATTERN } & \text { (memorization and repetition of language patterns) } \\ \text { F4. } & \text { DELAY } & \text { (not encouraging early language production) } \\ \text { F5. } & \text { MT } & \text { (using students' mother tongue for explanation) } \\ \text { F6. } & \text { TRANS } & \text { (using translation activities and exercises) } \\ \text { F7. } & \text { ERR PROD } & \text { (using tasks which allow error-laden production) } \\ \text { F8. } & \text { RULE SYN } & \text { (providing explicit rules of English syntax) } \\ \text { F9. } & \text { RULE PHON } & \text { (providing explicit rules of English phonology) } \\ \text { F10. } & \text { RULE VOCAB } & \text { (providing explicit definitions for new vocabulary items) } \\ \text { F11. } & \text { S GRAM CORR } & \text { (correction of grammatical errors in speech) } \\ \text { F12. } & \text { W GRAM CORR (correction of grammatical errors in writing) } \\ \text { F13. } & \text { PRONUN CORR (correction of pronunciation errors) }\end{array}$

Attitudes toward specific ESL methods and approaches

As can be seen above, the distinctive features which we employed characterize the "tenets" of many influential ESL methods and approaches. Our intention in decomposing these methods into a set 
of features was twofold: First we wanted a measure of teachers' attitudes toward specific practices which would be uninfluenced by the label to which these practices are typically attached. Second, we wanted an independent measure of whether there was any relationship between teachers' specific views about ESL practices and their general views about ESL methods.

To allow for this second comparison, we provided teachers with the names of 13 ESL methods and approaches. For each name, the teacher was asked to rate his or her opinion of it on a four-point scale ranging from very good to poor and also rate his/her familiarity with the method on a five-point scale.

The following methods and approaches were used: Grammar Translation, Audio-Lingual, Structural Global Audiovisual (S.G.A.V.), Notional-Functional, Cognitive Code, Total Physical Response, Suggestopaedia, Silent Way, Berlitz, Situational, Communicative, Community Language Learning (CLL), The Natural Approach.

\section{Attitudes toward the ordering and importance of language skills}

The final attitude measure obtained in the questionnaire concerned language skills. Teachers were asked to rate the relative importance of reading comprehension, listening comprehension, pronunciation, grammatical accuracy in speech, grammatical accuracy in writing, and spelling and punctuation on a five-point scale from very important to very unimportant. They were also asked to indicate the order in which the skills of reading, listening, writing and speaking should be introduced in an ESL course.

\section{Personal learning style}

The three sections above have outlined the nature of the attitude measurements which we obtained. As will be discussed below, most of the respondents had relatively strong views concerning both specific TESL practises and ESL methods. To what are these views related? A reasonable hypothesis is that teachers' views about how others should learn a second language are related to their own learning preferences. In other words, teachers "project" their learning styles onto their students. To investigate this possibility, we included the Learning Style Inventory developed by Kolb (1976) as part of the questionnaire. The Learning Style Inventory yields four learning style scores which allow respondents to be placed on a "learning style grid". On the basis of their grid placement, 
respondents may be characterized as convergers, divergers, assimilators, accommodators or balanced with respect to learning style. The significance of these styles and their relationship to teachers' attitudes will be discussed in Part 3 below. At this point, however, it is worth noting that of the 64 respondents, only 2 could be described as having a balanced learning style as measured by this technique.

\section{Characteristics of the teacher training course}

Sixty of the sixty-four respondents reported having participated in an ESL teacher training program or a course in language teaching methodology. It is often (although not always) the case that such courses seek to impart a set of methodological values. What then is the relationship between the views which were expressed as part of a teacher's TESL training and the views which he/she maintains as a practising ESL teacher?

The nature of this relationship was investigated in our study through a twenty-nine item subsection in which respondents were required to rate particular activities in terms of whether they were encouraged, discouraged, or not discussed in the teacher training program. The activities to be rated were matched by distinctive feature to those in the "techniques" subsection.

\section{Biographical data}

The final set of potential attitude correlates which we investigated concerned aspects of the teacher's individual biography. Teachers were asked to indicate their age, years of education and mother tongue. They were also asked to indicate whether they were competent in another language and whether they had ever learned a language in a classroom environment.

In addition, we were interested in the possibility that teachers' attitudes toward TESL techniques are influenced by their TESL experience-what they teach and whom they teach. We therefore included a set of questions that provided us with data concerning how long a teacher has taught ESL, what levels he/she has taught, what age groups, in which types of institutions and finally which methods and textbooks the teacher currently employs.

The questionnaire: summary

Our outline of the components of the questionnaire in the 
sections above has allowed us a discussion of the focus of this study. In short, the study asked: What do ESL teachers think and why? With respect to the second part of this question we have concentrated on a small set of variables which we hypothesized to be correlated to ESL teachers' attitudes. The intertwining of teachers' attitudes and the relationship between attitudes and learning style, teacher training and biographical variables is discussed in Part 3 of this report. This is preceded by a descriptive account of who our respondents are and what they think.

\section{PART 2: OVERALL TRENDS}

\section{The sample}

The questionnaires were distributed to all teachers of adult ESL in each of the five centres in our sample-102 teachers in total. Sixty-eight questionnaires were returned, of which four were incomplete and therefore dropped from the study. This yielded a total of 64 respondents, whose characteristics we outline in the sections below:

The majority of respondents (88\%) were native speakers of English and as can be seen in Figure 3, most were between the ages of 31 and 40. As is shown in Figure 4, the teachers in our sample had between 3 and 10 years of post secondary education.

\section{FIGURE 3}

Breakdown of the respondents by age group.

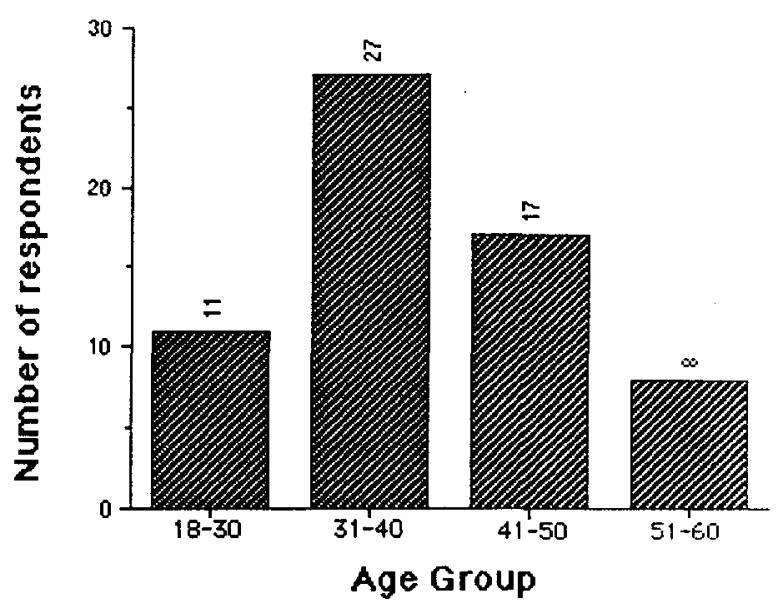


FIGURE 4

Breakdown of the respondents by years of secondary education.

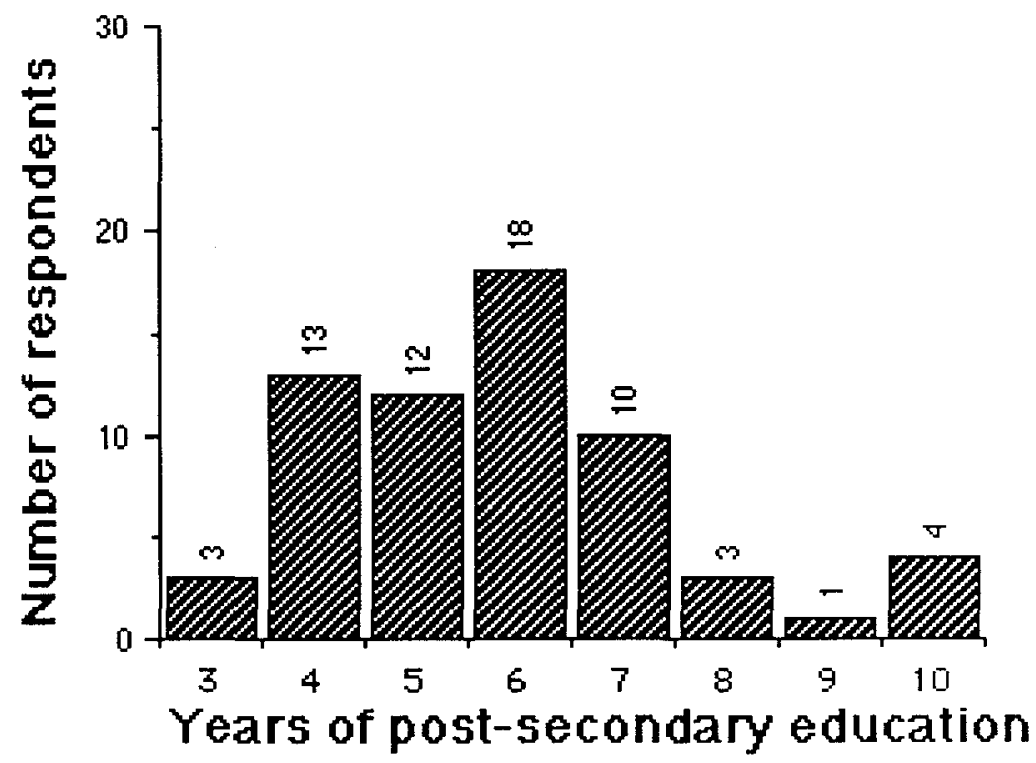

Eighty-seven percent of the teachers polled had learned at least one other language in a formal classroom environment and $94 \%$ had participated in a TESL course.

There was considerable diversity in the respondents' years of TESL experience (see Figure 5). As could be expected, TESL experience was correlated with age $(r=.53)$ such that the older teachers had been teaching ESL for more years than the younger teachers. On the whole, however, the group possessed a wide range of TESL experience. As has been noted above, 94\% had participated in a TESL course. In addition, $62 \%$ of the teachers had experience teaching all levels of ESL, $94 \%$ had taught more than one age group and $72 \%$ had taught in more than one type of educational institution (primary, secondary, continuing education, commercial, etc.).

It should be stressed at this point that although the subjects in our sample were drawn from four Canadian cities, they do not constitute a random sample of Canadian ESL teachers. Nevertheless we do not consider this sample to be unrepresentative. A series of one-way ANOVA's failed to reveal any significant differences among ESL Centres on any of the subject characteristics discussed above or any of the 13 attitude features in the questionnaire. We conclude, therefore, that the subjects in this sample were drawn 
from the same population and that our findings would generalize to other samples of adult ESL teachers in Canada.

FIGURE 5

The respondents' ESL teaching experience.

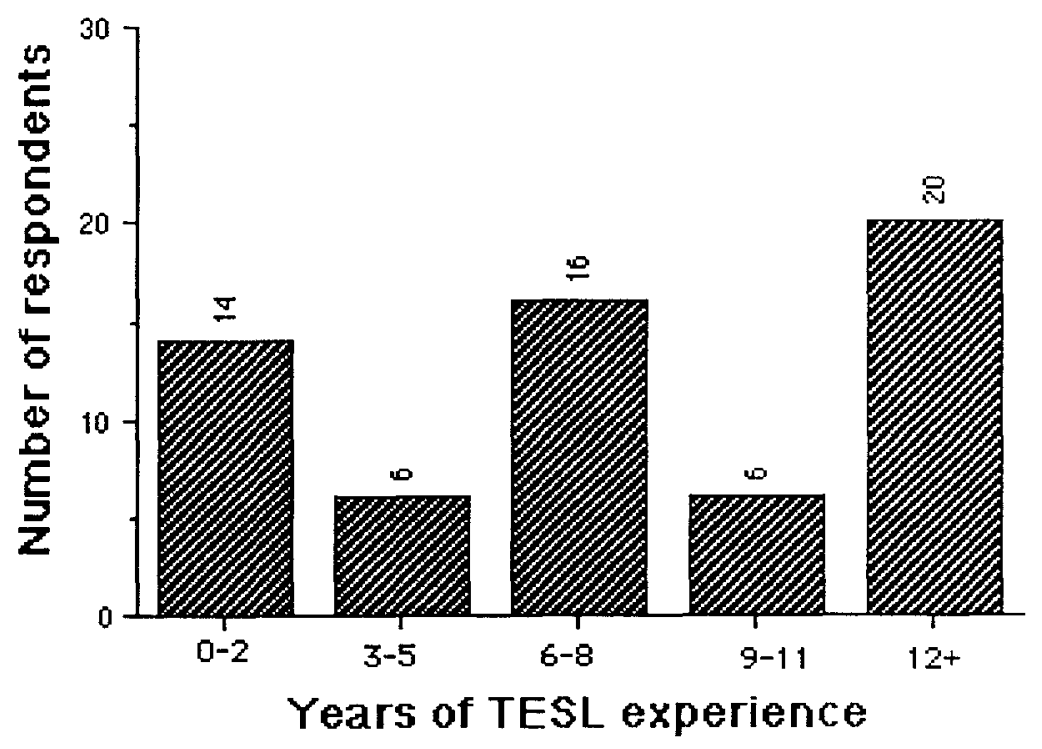

\section{The TESL attitudes}

The attitudes which the teachers expressed toward individual teaching methods are summarized in Figure 6. The respondents exhibited a marked preference for the communicative approach and a uniformly negative attitude toward the Grammar Translation approach. It should be noted (see Figure 7) that these were methods with which the respondents were quite familiar. Thus the simple and intuitively appealing hypothesis that teachers like the methods that they know best appears not to be correct. Indeed, there was only a weak correlation between method rating and method familiarity $(r=.34)$.

As far as the attitudes to TESL features are concerned, the respondents views were quite consistent with their ratings of ESL methods. Predominantly negative attitudes were expressed toward 
FIGURE 6

Teachers' attitudes toward ESL methods and approaches.

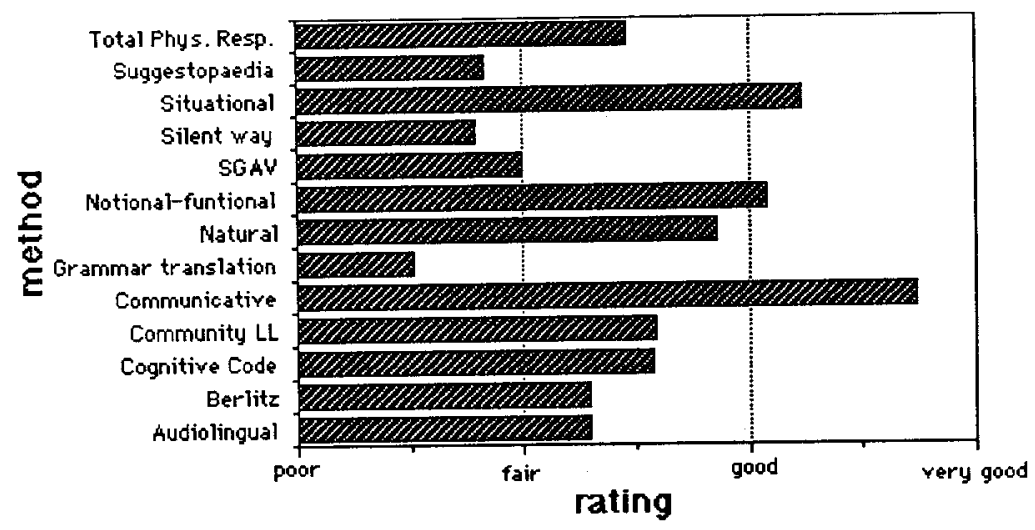

FIGURE 7

Teachers' familiarity with ESL methods and approaches.

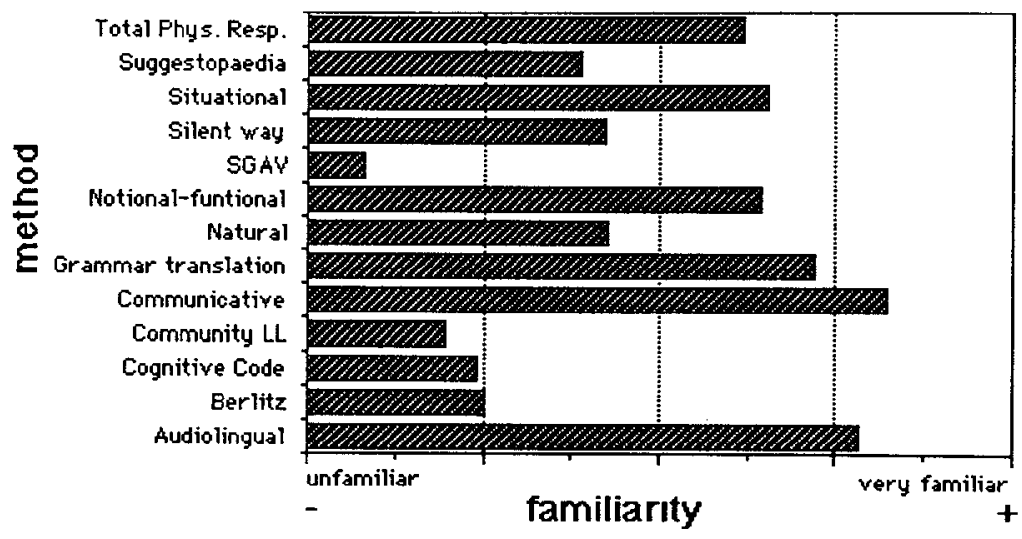

the use of translation and the use of the students' mother tongue in the ESL classroom. This is consistent with the negative rating of the Grammar Translation method. Teachers were of the general 
opinion that students should be given challenging exercises in which errors are likely to be made. In addition, they expressed the view that these errors should be corrected by the teacher. The respondents supported error correction in written syntax, spoken syntax and pronunciation. This consensus of opinion across language levels and modalities did not hold, however, for their views on providing explicit language instruction. Note in Figure 8, that the attitudes toward providing explicit rules of syntax and phonology, and providing explicit definitions for new words were quite different.

FIGURE 8

Teachers' attitudes toward TESL technique features.

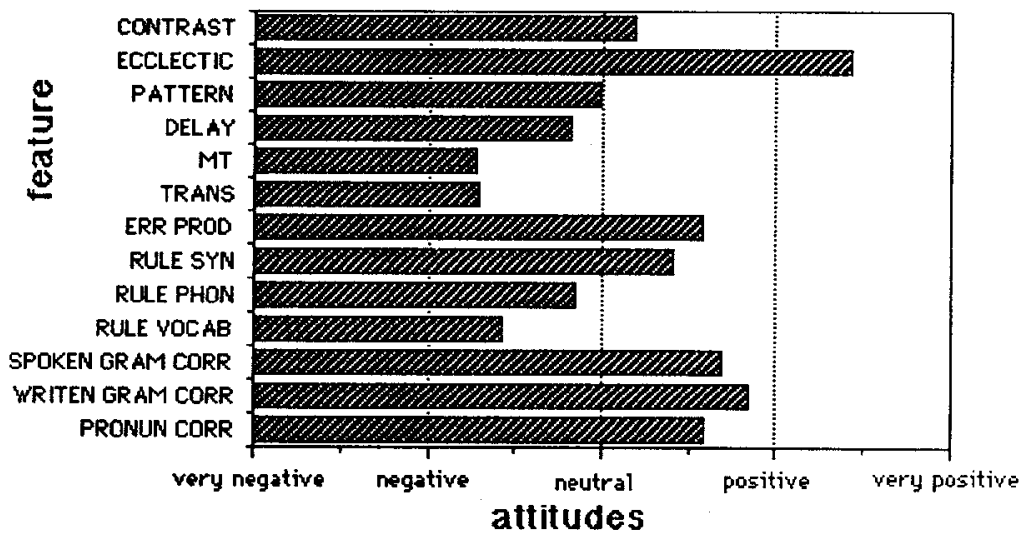

The teachers expressed a slightly negative attitude toward the feature DELAY. In other words, they did not approve of a period of "listening only" in the early stages of ESL instruction. This was consistent with their rating of the natural approach but somewhat at odds with their view that listening should precede speaking in an ESL syllabus. This latter datum was obtained from the section in which teachers are asked to propose an optimal ordering of skills in an ESL course. The ordering for the entire sample was:
(1) listening
(2) speaking
(3) reading
(4) writing

The dominance of listening was also evident in the respondents' ordering of skills according to their importance. As can be seen in Figure 9, listening is by far the most important skill, followed by pronunciation and reading comprehension. It is interesting that, in general, teachers appear to place a greater value on comprehension 
than on production. One possible explanation for this is that the comprehension skills were presented in the questionnaire as integrative skills whereas the production skills were broken down into semi-discrete components. It is conceivable that a production skill such as "making yourself understood" would have been given a relatively high importance ranking.

\section{FIGURE 9}

Teachers' ratings of the relative importance of language skills.

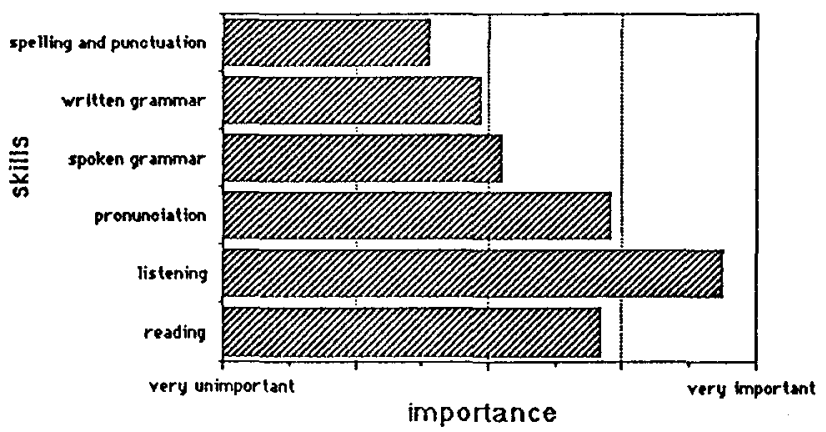

Finally, and perhaps most importantly, our respondents showed extremely favourable attitudes toward an eclectic methodological approach to teaching English and therefore very negative attitudes toward the use of any single TESL method. Teachers believe that effective ESL teaching requires a variety of techniques and that no single method or textbook can be relied upon (see Figure 2, Item 5). We take this finding to also indicate that teachers believe they have the expertise to determine the conditions under which any technique can be profitably employed. ${ }^{2}$

\section{PART 3:}

\section{TESL ATTITUDES AND TEACHER CHARACTERISTICS}

The data which we have summarized above represent the teachers' attitudes in terms of their central tendency across the entire sample. There was of course considerable variation among responses for attitudes toward TESL techniques and methods. Thus far, we have not discussed this variation in responses or the possible sources of this variation. The analysis of sources of variation is 
particularly important in light of our conclusion that our respondents feel that a self-determined teaching style is the best teaching style. As has been discussed in Part 1, our questionnaire also investigated factors (represented by ovals in Figure 1) which could be related to the composition of an individual's teaching style. In this section, the relationship of these factors to TESL attitudes is explored in some depth.

Our investigation of the effect of teacher characteristics employed the "feature scores" as the conceptual dependent variable. In cases where it was reasonable to dichotomize a teacher characteristic (e.g., whether they know another language) t-tests were employed for which each of the 13 attitude features served as dependent measures. In cases where the teacher characteristic was essentially categorical but not dichotomous (e.g., learning style), a series of one-way ANOVA's were conducted. Finally, in cases where the teacher characteristic could assume a wide range of values, the Pearson Product Moment Correlation Coefficient was used.

\section{Age, experience and education}

The first possibility that we investigated was whether TESL attitudes could be related to generational differences among respondents. This turned out to be the case for 4 of the features. There were significant effects of age for the features MT $(F(3,60)=2.684, p=.05)$, TRANS $(F(3,60)=3.179, p<.05)$, PRONUN CORR $(F(3,60)=3.86, p<.05)$, and W GRAM CORR $(F(3,60)=2.7$, $p=.05)$. For all of the features, post-hoc analyses revealed that it was the youngest group (18-30) which expressed the anomalous attitudes. Compared to the other age groups, young teachers showed the most positive attitudes toward the teacher's use of the students' mother tongue and toward translation exercises, but the most negative attitudes toward the correction of grammatical and pronunciation errors. These results were quite counter to our expectations. Our prediction was essentially that the younger teachers would lead in what are generally considered progressive TESL methods (e.g., exclusive use of the target language in the classroom). We investigated the possibility that the younger teachers were not as well trained as the older ones, but found no relationship between age and years of education $(r=14)$. The most reasonable explanation, then, remains that negative attitudes toward the use of the mother tongue in the classroom is related to actual TESL experience of which (as noted above) the younger teachers had less. 
Our further investigation of the manner in which years of teaching experience is related to TESL attitudes revealed a significant tendency of the more experienced teachers to concentrate on aspects of oral production. We divided our respondents into two groups: those with up to 5 years of TESL experience $(n=20)$ and those with more than 5 years of experience $(n=42)$. The more experienced teachers showed significantly more positive attitudes toward providing explicit rules of phonology $(p<.05)$, correcting pronunciation errors $(p<.01)$, and toward pattern practice $(p<.01)$. We find it noteworthy that these are the only features which showed significant differences between the two groups and that the more experienced teachers also showed a significantly higher rating of the importance of speaking in the "skill rating subsection" of the questionnaire.

\section{Methods used and students taught}

Recall that the teaching style questionnaire polled teachers' current teaching situation with respect to whether they taught according to a particular method and whether they taught linguistically homogeneous or heterogeneous groups of students. Although most teachers expressed positive attitudes toward the individual TESL methods they employed, we found no relationship between methods currently used and general TESL attitudes. However, when we investigated the differences between teachers who report using a method $(n=31)$ and teachers who report using no method at all $(n=33)$, significant effects were found. Teachers who make up their own method, appear to be much more inclined to employ "metalinguistic instruction". They showed more positive attitudes toward explicit instruction in all domains $(p<.01)$ and also more positive attitudes toward the feature CONTRAST ( $t$ $(62)=4.07, p<.001)$. It appears then that this class of teachers, who by choice or circumstance do not use a packaged method, tend to want to teach their students much more about the target language than their colleagues do.

We expected to find a strong relationship between teachers' attitudes toward the features MT and TRANS and whether they had taught a group of students who all have the same mother tongue. This prediction was not supported. Instead, we found that those teachers who taught a linguistically homogeneous group $(n=12)$ showed significantly more negative attitudes toward the correction of syntactic errors in speech $(t(62)=3.53, p<.001)$ and in writing $(t$ $(62)=2.55, p<.05)$ as well as more negative attitudes toward 
providing explicit rules of syntax $(t(62)=4.4, p<.001)$.

With respect to other aspects of teachers' biographies, we found no relationship between teachers attitudes and their total years of education, the number of other languages they reported knowing, or the number of other languages they had learned in a formal environment. Indeed, as we have noted above, those significant effects which we did find, were rarely in accordance with our expectations. These effects were, however, neither random nor isolated. Rather, they tended to emerge in clusters which were organized around particular themes. The relevant theme for teachers who have taught linguistically homogeneous classes, for example, is clearly the irrelevance of syntax. One possible explanation for this de-emphasis may be that in cases where classes are homogeneous (e.g., ESL classes in Quebec), teachers may see themselves as offering what students cannot get outside the classroom-the opportunity for interaction with a native speaker. They therefore concentrate on communication rather than structure.

\section{Teacher's learning style}

The last teacher characteristic which we investigated was the personal learning style of the teacher. In accordance with the procedure provided in Kolb (1976) we assigned to teachers one of four learning styles. These styles, which are described immediately below, are labeled converger, diverger, assimilator and accommodator.

The converger learning style is characteristic of individuals whose greatest strength lies in the practical application of ideas. According to Kolb, this type of learner is relatively unemotional and prefers to deal with things rather than people.

Divergers have the opposite learning strengths of the converger. They are interested in people and tend to be imaginative and emotional. The diverger excels in the ability to view concrete situations from many perspectives.

The assimilator's greatest strength lies in the ability to create theoretical models. This person excels in inductive reasoning and in assimilating disparate observations into an integrated explanation. Like the converger, this type of person is more interested in abstract concepts than in people but unlike the converger, the assimilator tends to be little concerned with the practical application of ideas.

Finally, the accommodator's greatest strength lies in doing things, getting involved and taking risks. The accommodator has an "intuitive" approach to problem solving and tends to be social and 
action-oriented.

Our sample contained 10 convergers, 15 divergers, 9 assimilators and 16 accommodators. As we have noted above, 2 of the teachers were balanced with respect to learning style and an additional 12 fell on the border between two learning styles. These teachers were not included in the learning style analysis. Thus our sample of teachers with clearly definable learning styles consisted of 50 respondents. It is perhaps noteworthy at the outset that accommodators and divergers make up $62 \%$ of this sample. There was thus a slight tendency for the teachers to have concrete-active learning styles as opposed to the abstract-reflective styles of the convergers and assimilators.

We expected significant effects of learning style for those features which expressed a psychological rather than a linguistic orientation. For example, accommodators, because they are "intuitive", should be less inclined to provide explicit rules (of either syntax or phonology) and should be more likely to favour pattern practice because it is an "action-oriented" and non-reflective TESL technique. Divergers and assimilators, on the other hand, should assign the activity of pattern practice a very low rating because they, as students, would find it uninteresting.

As it turned out, three features showed significant effects of learning style. These were PATTERN $(F(3,46)=3.495, p<.05)$, ERR PROD $(F(3,46)=3.124, p<.05)$ and RULE PHON $(F(3,46)=3.684, p<.01)$. In all cases, however, the post-hoc analysis revealed the differences between learning styles to be quite different from our expectations. The assimilators, the most abstract non-applied group, showed significantly more positive attitudes toward pattern practice. The divergers showed the most favourable attitudes toward providing rules of phonology and the accommodators showed the most favourable attitudes toward the feature ERROR PROD.

Again we find these results surprising, but not unsystematic. Apparently, our straight-forward hypothesis that teachers project their learning styles onto their students was incorrect. Rather, it seems that we found an indication that teachers either compensate for their learning styles or use the ESL classroom to act in a fashion that they perceive to be contrary to their nature. We see this latter hypothesis as particularly intriguing. There is considerable anecdotal evidence that many teachers find TESL rewarding precisely because it offers them the opportunity to do what does not come naturally. Thus for introverted teachers, it is the opportunity for social contact and for "intuitive thinkers" it is the opportunity to 
explore the systematicity of syntax and phonology.

These views are of course speculative, but we see this phenomenon of "persona reversal" as a fruitful area for further research with more elaborate learning style and cognitive style batteries.

\section{Teacher characteristics: summary}

Our investigation of the relationship between teacher characteristics and TESL attitudes has, in our estimation, revealed some interesting effects. The data support the hypothesis that a teacher's age, amount and type of TESL experience, and individual learning style are all related to the attitudes that the teacher has toward TESL practices. In all cases, however, we found that the effects were localized to specific features or clusters of features-there were no "across the board" effects. Thus we are forced to conclude that although some characteristics of the teacher do make a difference, they do not provide us with an account or an explanation of the overall pattern of TESL attitudes that is displayed in Figure 8. We maintain the view, however, that Figure 8 represents an internally consistent picture of TESL attitudes. In the section below, we report on our investigation of the relationship between this attitude picture and the TESL attitudes which were promoted as part of the respondents' TESL training courses.

\section{Teacher training}

Our first observation in the investigation of this factor was the marked lack of variability in the teachers' accounts of which activities were encouraged and discouraged in their TESL training courses. In the case of the feature MT, for example, there was complete unanimity. In every case the teacher-training course taught that the use of the students' mother tongue should be avoided. These data raise the question: What is the source of the attitudinal homogeneity that inclines TESL teachers to impart a relatively unified set of values to their teacher-trainees?

Before we address this question, it is necessary to report on the relationship we found between the teacher-training course and the teachers' attitudes. As has been noted above, the teacher-training section of the questionnaire was organized around the same set of features as the attitude section (except for the features W GRAM CORR and DEL PROD). We analyzed the correlation between the average teacher training attitude scores and the average TESL attitude scores which were presented in Figure 8. The correlation 
between these two variables was .85 which was by far the strongest relationship between the attitudes and any other variable, accounting for almost three quarters of the variance $\left(r^{2}=.7225\right)$.

We are left now with two possible conclusions: The first is that TESL methodology courses play a major role in determining ESL teachers' views about how ESL should be taught. The second is that both ESL teachers and TESL teachers in Canada are strongly influenced by a TESL "Zeitgeist" ${ }^{n}{ }^{3}$ Note that in either case we are suggesting that the freedom of opinion that an ESL teacher perceived himself/herself to have is largely illusory.

In our view, the second conclusion is preferable to the first because it offers a simultaneous account of the training attitudes, the teaching attitudes and the relationship between them. It does not, of course, offer an account of the source and nature of the Zeitgeist. We will consider this issue in the final section below.

\section{PART 4: CONCLUSIONS AND SPECULATIONS}

Our findings with respect to Canadian ESL teachers' attitudes toward TESL techniques have been somewhat paradoxical. On the one hand, the responses for the feature ECLECTIC, reveal that teachers feel strongly that their activities in the classroom should be self-determined. In other words, they shouldn't be told how to teach but rather should be free to teach in accordance with their individual views. On the other hand, it just happens to be the case that ESL teachers have very similar views and "choose" to teach very much as they were told to teach.

Of course, as we have noted above, there is individual variation which to some extent can be accounted for by a set of subject variables such as age, experience and teaching environment. However, this variation must be seen against the backdrop of the set of shared values and beliefs represented in Figure 8. In our view, this figure offers a snapshot of what might be the current state of TESL Culture in Canada. ${ }^{4}$ We use the term "culture" here in its technical anthropological-sociological sense as knowledge, norms, values and beliefs shared by members of a community (Tylor 1873; Rohner 1984).

Cultural norms and attitudes have properties which we consider very important to the understanding of the responses which we obtained-namely that they are both interpersonal and intrapersonal. On the one hand, these attitudes are the products of interpersonal communication and tend to acquire "a life of their own" of which no member of the culture is in control. On the other 
hand, they are internalized by each member so that each member considers the shared attitudes to be their personal attitudes.

As a (non-TESL) example of this phenomenon, consider the change in North American attitudes toward smoking and recycling during the last decade. It seems now almost inconceivable that 100 millimeter cigarettes were once signs of sophistication and disposable containers signs of modernity. It is probably safe to assume that a current poll of attitudes to these products would reveal a culture-wide change of view. However, the people polled would believe that they are expressing views which are products of their own conviction.

It seems to us that our questionnaire revealed the same type of phenomenon. The professionalization of the field which we noted at the outset of this paper has clearly led to a level of interpersonal communication which facilitates the circulation of attitudes. These attitudes make up a TESL Zeitgeist which as a result of its omnipresence affects every member of the community. In some cases it determines what teachers think they believe-in all cases it sets the agenda for what is worth discussing.

Because our questionnaire concentrated on fairly broad categories of TESL methodology there are many other aspects of this Zeitgeist which we were unable to investigate. Those we did investigate show a unified picture which we present below in the form of a narrative. The material in brackets refers the reader to the relevant figures or data from which the corresponding sentence derives:

Language is a system of communication which is best taught through communication (Figure 7). Of greatest importance in the acquisition of English as a second language is the ability to extract meaning from spoken English (Figure 9). Thus ESL courses should begin by developing this skill.

A good ESL teacher should employ a variety of TESL techniques and adjust them to suit the circumstances (ECLECTIC). Students should be encouraged to take risks in expressing their ideas (ERR PROD) and teachers should support this risk-taking by offering constructive error correction when appropriate (CORR). A teacher can also help the student by pointing out differences and similarities between English and the student's mother tongue (CONTRAST) and by supplying rules of English grammar (RULE SYN) and (less profitably) English pronunciation (RULE PHON). Although the teacher's explanations may help the student, it is important to try to ensure that the process of second language acquisition be as natural as possible (Figure 7). For example, word 
meanings should be acquired in context through target language associations (Fig. 7, Sit.; Fig. 8 RULE VOCAB) and as far as possible, English should be the sole language of communication and explanation in the classroom (Fig. 8, MT, TRANS).

We note in conclusion that the above narrative represents an approach to TESL that the authors consider intuitively appealing. Moreover, we find that the narrative expresses many of our views of effective ESL teaching practices. If these are also your views, the question to ask is: Where did they come from?

\section{NOTES}

1. We wish to thank the following administrators and their staff for their cooperation in this study: Roselie Banko, University of Alberta, Edmonton Alberta; David Dom and David Wood, Mount Royal College, Calgary Alberta; Grace Mathews and Irving Rabinovitch, Dept. of Adult Education, The Protestant School Board of Greater Montreal, Montreal Quebec; Richard Moon, St. Lawrence College, Cornwall, Ontario. We also thank Mary Kanik for her assistance in the preparation of the data and Lorna Rowsell for commenting on an earlier draft of this paper.

2. It is conceivable that an administrator might dictate that a particular array of books be used. In this case the teacher would be teaching eclectically but would not be exercising any freedom of approach. We assume that although such situations occur, they are not what our respondents had in mind.

3. Zeitgeist is a German sociological term which can be roughly translated as "spirit of the times".

4. We strongly suspect that the culture to which we refer is not specifically Canadian. However, because our sample was drawn exclusively from ESL Centres in Canada, we don't feel justified in generalizing beyond this population. 


\section{THE AUTHORS}

Gary Libben is an Assistant Professor in the Department of Linguistics, The University of Calgary. His areas of research are second language acquisition, neurolinguistics and psycholinguistics.

Tammi Rossman-Benjamin holds an M.A. in Applied Linguistics from Concordia University and an M.A. in Experimental Psychology from the University of Pennsylvania. She has ESL teaching experience in both Canada and Israel.

\section{REFERENCES}

Kolb, D. A., Rubin, I. W. \& McIntyre, J. M. (1984). Organizational psychology: An experiential approach to organizational behavior. Englewood Cliffs, NJ: Prentice-Hall.

Larsen-Freeman, D. (1986). Techniques and principles in language teaching. New York: Oxford University Press.

Richards, J. C. \& Rodgers, T. S. (1986). Approaches and methods in language teaching. New York: Cambridge University Press.

Rohner, R. P. (1984). Toward a conception of culture for cross-cultural psychology. Journal of Cross-Cultural Psychology, $15,11-138$.

Tylor, E. B. (1873). Primitive culture: Researches into the Development of Mythology, Philosophy, Religion, Language, Art and Custom. London: John Murray. 